\title{
Protection de sites portuaires par des ouvrages flottants en béton
}

\author{
Yann Renoul $^{(\mathrm{a})}$, Jean Bougis ${ }^{(\mathrm{b})}$ \\ ${ }^{(a)}$ Ingénieur, SOGREAH CONSULTANTS, 11, Rue J.P. Boullé, BP 93, 56303 Pontivy Cedex, \\ Tél. : 02.97.25.38.50, \\ yann.renoul@sogreah.fr \\ (b)Ingénieur Conseil, 34 Chemin du Moulin, 06650 Opio, \\ Tél. : 04.93.77.74.22, \\ jbougis@aws.fr
}

\section{Résumé:}

Le recours de plus en plus fréquent à des brise-lames flottants pour protéger des ports de plaisance toujours plus exposés impose la réalisation d'études de conception complexes. Les points clés de la méthodologie et du savoir-faire développés par les deux bureaux d'études pour maîtriser les dispositions constructives et les moyens d'études des brise-lames flottants en béton sont exposés et illustrés par des exemples empruntés aux nombreuses études de projet ou d'exécution réalisées en commun. Une attention particulière est apportée aux aspects dynamiques du fonctionnement de ces brise-lames et à leurs conséquences sur les efforts à transmettre aux ancrages et sur les efforts internes à la structure.

\section{Abstract:}

The more frequently use of floating breakwaters in order to protect pleasure harbours which are more and more exposed impose the realization of complex design studies. The most important points of methodology and know-how developed by the two engineering to obtain the mastership of constructive arrangements and of study means of concrete floating breakwaters are exposed and illustrated by examples proceeded from numerous projects and executives studies realized in common. A particular attention is paid to dynamics aspects of these breakwaters running and to theirs consequences on the loads which must be transferred to the mooring systems and on the internal loads of the structure.

Mots-clés : brise-lames flottant, ponton lourd, ponton béton, ancrage, pieux.

Keywords: floating breakwater, pontoon, concrete pontoon, anchorage, piles.

\section{Introduction}

Les contraintes environnementales et réglementaires limitent désormais l'utilisation d'ouvrages fixes de génie civil pour protéger les extensions des ports de plaisance. Le recours à des solutions flottantes s'impose donc quand les conditions d'agitation le permettent. Les pontons flottants représentent une classe importante de brise-lames qui, à défaut d'être la plus efficace, est en tout cas la plus répandue. Ils sont maintenus en place soit par des lignes d'ancrage, soit par des pieux. Ces systèmes d'ancrage présentent des caractéristiques de comportement radicalement différentes qui ont une grande influence sur le résultat obtenu.

La protection d'un plan d'eau contre la houle pose de nombreuses questions liées, d'une part à l'agitation et aux performances d'atténuation des brise-lames, et d'autre part à la résistance des brise-lames aux efforts dus à la houle. Ceux-ci gouvernent le dimensionnement des ancrages et des liaisons entre les différents éléments d'une panne. La réussite d'un projet de brise-lames flottants en béton est subordonnée à l'adoption de dispositions constructives 
adaptées et à la maîtrise de méthodes de calculs complexes. Les problèmes rencontrés lors du développement empirique d'installations flottantes (mauvaises performances, ruptures d'ancrages) et l'exposition toujours plus sévère des plans d'eau incitent les maîtres d'ouvrage à intégrer aux maîtrises d'œuvre et aux exécutions des études de plus en plus complètes pour les brise-lames flottants. Les nombreuses études de projet et d'exécution réalisées depuis huit ans ont conduit les deux bureaux d'études à développer une méthodologie et un savoir faire, dont les principaux points sont exposés ici.

\section{Dimensionnement fonctionnel de l'ouvrage}

Le dimensionnement fonctionnel de l'ouvrage impose les choix technologiques fondamentaux du système, de ses liaisons et de ses ancrages puis la géométrie du flotteur et la raideur de ses ancrages pour qu'il puisse assurer la protection désirée du plan d'eau. La largeur et le tirant d'eau dépendent de l'agitation à atténuer (période et hauteur) qui gouverne l'excitation hydrodynamique du flotteur, et des périodes propres du système qui gouvernent sa réponse dynamique à cette excitation. Cette démarche est guidée et contrôlée par l'étude d'agitation. Pour les plus gros brise-lames, une grande attention doit être portée à la génération d'harmoniques qui peut augmenter l'énergie véhiculée par les ondes courtes susceptibles d'exciter les modes propres des bateaux de plaisance ou des appontements.

\subsection{Choix technologiques de l'ouvrage et de ses ancrages}

Pour s'opposer à la transmission des lames et de la quantité de mouvement qu'elles véhiculent, il est nécessaire de reprendre des efforts importants et de les transmettre le plus directement possible au sol en limitant les mouvements de l'ouvrage. Les meilleures performances spécifiques vont ainsi de paire avec la rigidité des pannes et de leurs ancrages. Les ancrages sur pieux sont donc les plus efficaces. Ils éliminent aussi les problèmes liés aux déplacements horizontaux des brise-lames flottants ; lesquels peuvent devenir importants avec des ancrages sur lignes, surtout dans les sites à fort marnage. Les choix de rigidité ne portent pas sur les flotteurs eux-mêmes, mais sur l'ensemble composé d'une succession de flotteurs. La recherche d'une forte rigidité longitudinale de la panne conduit à privilégier les systèmes d'assemblages semi-rigides. Un élément est ancré sur deux pieux et les autres sont liés à leurs deux voisins et à un pieu. Il y a un pieu de plus que d'éléments. Les efforts de liaison entre éléments peuvent être très importants et il n'est plus intéressant d'y recourir quand l'agitation impose un ouvrage de forte section ou lorsque les raideurs des pieux sont très hétérogènes (sol hétérogène, forte pente du substratum). Chaque élément doit alors être isolé sur deux pieux.

\subsection{Choix de la largeur des brise-lames}

Dans une profondeur $h$, une houle de période $T$ et de longueur d'onde $\lambda$ exerce sur une structure des efforts d'excitation qui dépendent, dans une large mesure, de la période. L'expérience (Vugts ${ }^{1}$ ) montre que pour un flotteur de section rectangulaire de longueur $L$, de largeur $B$ et de tirant d'eau $D$ :

- Lorsque $\lambda$ est petite devant $B$, les effets des multiples crêtes et creux se compensent ; les efforts d'excitation restent faibles tant que : $\lambda / B<1.6$.

- Lorsque $\lambda$ est grande devant $B$, le flotteur bouchonne ; les efforts d'excitation sont importants et indépendants de la période dès que : $5.6<\lambda / B$.

- Entre les deux, les efforts dépendent de $T$ en passant ou non par un maximum.

Un flotteur à section rectangulaire peut donc s'opposer aisément à la transmission de la houle jusqu'à des conditions intermédiaires, $\lambda<2 B \sim 3 B$, tant que les efforts d'excitation restent faibles. Au-delà les choses se compliquent nécessairement. La figure 1 présente les coefficients de transmission de pontons flottants de section rectangulaire en fonction de $\lambda / B$ 
$(1.25<h / B<2.0,3.3<B / D<10)$ issus d'essais en canal (E) Bougis $^{2}$, Carver $^{3,4}$, et Blumberg $\& \operatorname{Cox}^{5}$ ) et de calculs 2D $(\mathrm{C})$ réalisés avec le logiciel Poséidon (Bougis ${ }^{6}$ ). La courbe ajustée est également tracée.

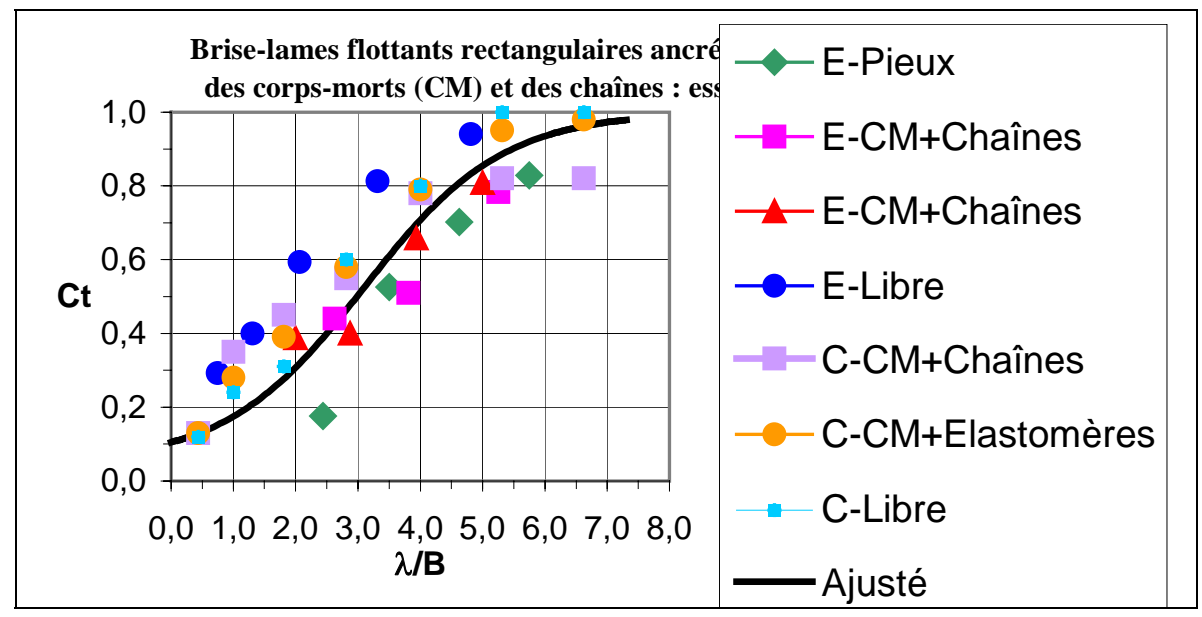

Figure 1: Coefficient de transmission de brise-lames rectangulaires

\subsection{Choix du tirant d'eau des brise-lames}

L'efficacité d'un brise-lames flottant croît avec les raideurs horizontales de ses ancrages. Les brise-lames sur pieux sont plus efficaces que ceux sur lignes caténaires ou élastomères ; les brise-lames libres sont les moins efficaces (figure 1). La théorie montre que l'efficacité d'un brise-lames dépend essentiellement de ses six périodes propres (rapport entre les masses et les raideurs). Les mouvements oscillatoires s'entretenant par échanges entre l'énergie cinétique et une énergie potentielle, il faut donc distinguer deux groupes de modes propres :

- Ceux liés à l'énergie potentielle de gravité. Engendrés par un rappel hydrostatique, ils concernent les mouvements à composante verticale (pilonnement, roulis, tangage) et ne dépendent que des formes de carène et du centre de gravité.

- Ceux liés à l'énergie potentielle élastique. Engendrés par un rappel élastique, ils concernent tous les mouvements entravés par un ancrage ou par une liaison entre les éléments et ne dépendent que de la nature de la liaison.

Pour une structure flottante, les modes propres "hydrostatiques" existent indépendamment des liaisons, mais sont modifiés par ces liaisons. Pour des périodes d'oscillations en deçà des périodes hydrostatiques, le comportement du flotteur est de type inertiel ; au delà, il est de type hydrostatique. Au premier ordre, les trois périodes propres hydrostatiques des flotteurs parallélépipédiques, ne dépendent que du tirant d'eau sous la forme $T \approx 3 \sqrt{D}$. Cette période coïncide avec celle liée au rapport $\lambda / B$ dans la fourchette $3.5<B / D<4.0$ qui correspond au minimum d'amortissement d'onde de gravité en roulis, c'est-à-dire au minimum de génération, et donc de transmission, des vagues par le mouvement de roulis.

\subsection{Choix des autres paramètres des brise-lames}

Les autres paramètres doivent également être fixés lors de la conception :

- Le franc bord : Pour limiter les franchissements, il doit être au moins de l'ordre de $80 \%$ de la hauteur significative.

- La longueur des éléments : L'efficacité du brise-lames croît avec l'allongement $L / B$ de ses éléments. La longueur est limitée par le procédé de construction, les moyens de levage et les ancrages (efforts et raideur). 
- L'espacement entre éléments : Pour des ancrages sur pieux, il peut être très faible. Pour des ancrages sur lignes, il relève de deux considérations contradictoires : éviter les risques de collisions entre éléments et éviter les transmissions de houles par effets de bord. L'espacement optimal est voisin de $B / 3$.

\section{$\underline{\text { 3.Dimensionnement structurel de l'ouvrage }}$}

\subsection{Efforts d'ancrages}

Les efforts dimensionnant pour les brise-lames flottants sont essentiellement dus à la houle et aux vagues d'étraves. Les efforts dus au vent et au courant peuvent l'être aussi, en zones exposées, pour des pannes servant d'appontement permanent. La tenue à l'accostage, normal et accidentel, doit alors être étudiée.

\subsubsection{Efforts engendrés par la houle}

En désignant par $M$ la masse du flotteur, $M_{a}$ sa masse d'eau ajoutée, $A_{g}$ son amortissement d'onde de gravité, $A_{v}$ son amortissement visqueux, $H$ sa raideur hydrostatique, $K$ la raideur de son ancrage, $F_{e}$ la force d'excitation de la houle, l'équation de la mécanique d'un corps flottant à un seul degré de liberté s'écrit :

$$
\left(M+M_{a}\right) \ddot{X}+\left(A_{g}+A_{v}\right) \dot{X}+(H+K) X=F_{e}
$$

Pour un mouvement $X$ sinusoïdal à la pulsation $\omega$, cette équation devient :

$\left[-\omega^{2}\left(M+M_{a}\right)-i \omega\left(A_{g}+A_{v}\right)+(H+K)\right] X=f_{e}$

avec : $X=\mathfrak{R}\{x \exp (-i \omega t)\}$ et $F_{D}=\mathfrak{R}\left\{f_{e} \exp (-i \omega t)\right\}$ où $: x$ et $f_{e}$ sont complexes. L'effort d'ancrage $f_{k}=-K x$ s'écrit donc en négligeant les amortissements :

$$
f_{k} \approx-\frac{K}{H+K-\omega^{2}\left(M+M_{a}\right)} f_{e}=-\left[1-\frac{H-\omega^{2}\left(M+M_{a}\right)}{K+H-\omega^{2}\left(M+M_{a}\right)}\right] f_{e}
$$

Pour le mouvement d'embardée la raideur hydrostatique est nulle : $H=0$. Il reste :

$$
f_{k} \approx-\left[1-\frac{-\omega^{2}\left(M+M_{a}\right)}{K-\omega^{2}\left(M+M_{a}\right)}\right] f_{e}=-\left[\frac{\omega_{n}^{2}}{\omega_{n}^{2}-\omega^{2}}\right] f_{e}=-\left[\frac{T^{2}}{T^{2}-T_{n}^{2}}\right] f_{e}
$$

où $T_{n}=2 \pi / \omega_{n}$ est la période propre d'embardée. Cette formulation montre que :

- La raideur de l'ancrage $K$ intervient de manière fondamentale dans l'effort d'ancrage qui s'annule, bien évidemment, avec la raideur.

- A la résonance de l'ancrage, seul l'amortissement permet de limiter cet effort. L'amortissement visqueux ne peut alors plus être négligé.

- Quand la raideur tend vers l'infini, l'effort d'ancrage tend vers : $f \approx-f_{e}$. La pulsation propre tend alors vers l'infini tandis que la période propre s'annule.

\subsubsection{Evaluation des périodes propres}

A la vue de l'équation (4), il est clair que les périodes propres du brise-lames ne gouvernent pas seulement sa capacité à atténuer la houle, mais aussi ses efforts d'ancrage et de liaisons et qu'une attention particulière doit leur être portée. En général, les termes de masse effective des pieux et des liaisons ne représentent que 3\% à 5\% des termes de masse des 
flotteurs, les périodes propres peuvent donc être évaluées en ne tenant compte de l'ancrage que par ses raideurs. Compte tenu des dissymétries des ancrages sur pieux, il est indispensable d'effectuer le calcul des périodes propres à partir des matrices complètes en tenant compte de tous les couplages entre les degrés de liberté. Ne retenir que les termes diagonaux conduit à de grossières erreurs. L'algorithme du QZ qui permet d'obtenir les modes propres d'un système libre pouvant posséder des valeurs propres nulles ou infinies, est particulièrement bien adapté à cet effet. Les vecteurs propres sont ensuite calculés par l'algorithme du QU de Householder. Ils permettent d'associer les mouvements propres des pontons aux périodes précédemment calculées. Le tableau 1 présente les périodes propres d'un ensemble de deux pontons ancrés sur trois pieux et liés par une liaison semi-rigide. Les degrés de liberté sont indiqués par leurs initiales. Il est clair que l'approximation n'est pas acceptable !

Tableau 1 : Périodes propres d'un brise-lames avec ses liaisons (Anse du Ter) cavalement $C$, embardée $E$, pilonnement $P$, roulis $R$, tangage $T$ et lacet $L$.

\begin{tabular}{|c|c|c|c|c|c|c|c|c|c|c|c|c|c|}
\hline Sans & $\mathrm{T}(\mathrm{s})$ & 0.54 & 0.58 & 0.69 & 0.75 & 1.04 & 1.19 & 1.22 & 1.24 & 1.91 & 1.92 & 2.10 & 2.14 \\
couplages & $\mathrm{ddl}$ & $\mathrm{C}$ & $\mathrm{C}$ & $\mathrm{L}$ & $\mathrm{L}$ & $\mathrm{E}$ & $\mathrm{E}$ & $\mathrm{T}$ & $\mathrm{T}$ & $\mathrm{P}$ & $\mathrm{P}$ & $\mathrm{R}$ & $\mathrm{R}$ \\
\hline Avec & $\mathrm{T}(\mathrm{s})$ & 0.42 & 1.14 & 1.79 & 0.53 & 0.97 & 1.23 & 3.07 & 0.80 & 3.10 & 3.11 & 1.63 & 6.90 \\
Couplages & ddl & $\mathrm{C}$ & $\mathrm{CE}$ & $\mathrm{ER}$ & $\mathrm{ECL}$ & $\mathrm{ECL}$ & $\mathrm{ECR}$ & $\mathrm{P}$ & $\mathrm{PT}$ & $\mathrm{P}$ & $\mathrm{PT}$ & $\mathrm{ER}$ & $\mathrm{RE}$ \\
\hline
\end{tabular}

Dans les sites à forts marnages, il est indispensable d'effectuer un calcul à marée basse et un calcul à marée haute et de s'assurer que l'évolution des modes propres ne présente pas de risque pour les niveaux intermédiaires.

\subsubsection{Dimensionnement des pieux}

Un pieu à inertie constante est caractérisé par quatre paramètres : son diamètre $d$, son épaisseur $e$, sa longueur $l$ dont sa longueur fichée dans le sol $f$. Son dimensionnement est le fruit d'un compromis entre quatre types de contraintes :

- La géométrie du collier de liaison avec le pieu impose une gamme de diamètres et d'épaisseurs minimales pour éviter l'ovalisation du pieu.

- Les efforts subits par le pieu imposent des couples $(d-e)$ pour reprendre les efforts avec une épaisseur minimale pour éviter le cloquage de la peau du pieu.

- La tenue du pieu dans le sol impose des couples $(d-f)$.

- Le brise-lames ne doit pas avoir de périodes propres énergétiques dans la gamme des périodes d'excitation par la houle.

Pour un ponton de forte section en grande profondeur soumis à un clapot de période assez grande ( $T \sim 4$ à $4.5 \mathrm{~s})$ avec une hauteur assez faible $(H<1 \mathrm{~m})$, le critère dimensionnant n'est plus lié aux efforts, mais à la raideur des pieux. La dernière contrainte montre aussi que les ancrages sur lignes souples sont à réserver aux cas de clapots de petites périodes et de faibles hauteurs lorsque les périodes propres peuvent être supérieures aux périodes d'excitation. Le tableau 2 donne des efforts alternatifs maximaux et les efforts de dérive sur houle pour cinq brise-lames.

Tableau 2: Efforts dimensionnants de différents brise-lames flottants

\begin{tabular}{|c|c|c|c|c|c|c|c|c|}
\hline Site & $\begin{array}{c}\text { Nombre } \\
\text { Eléments }\end{array}$ & $\begin{array}{c}L \\
\mathrm{M}\end{array}$ & $\begin{array}{c}B \\
\mathrm{~m}\end{array}$ & $\begin{array}{c}D \\
\mathrm{~m}\end{array}$ & $\begin{array}{c}H_{\mathrm{s}} \\
\mathrm{m}\end{array}$ & $\begin{array}{c}T_{\mathrm{p}} \\
\mathrm{s}\end{array}$ & $\begin{array}{c}F_{\text {alt.max }} \\
\mathrm{kN} / \mathrm{m}\end{array}$ & $\begin{array}{c}F_{\text {moy }} \\
\mathrm{kN} / \mathrm{m}\end{array}$ \\
\hline Anse du Ter & 2 & 60 & 4.0 & 1.00 & 1.2 & 2.8 & 28.1 & 0.7 \\
Driasker & 2 & 44 & 3.76 & 1.03 & 0.5 & 2.0 & 28.9 & 0.1 \\
Moulin Blanc & 3 & 90 & 5.0 & 1.10 & 1.2 & 4.0 & 27.2 & 0.7 \\
Ste Catherine & 2 & 62 & 5.0 & 1.25 & 0.85 & 2.8 & 20.8 & 0.5 \\
Ste Catherine & 5 & 129 & 5.0 & 1.25 & 0.65 & 2.4 & 13.1 & 0.5 \\
\hline
\end{tabular}


Ainsi, la conception de l'ancrage est un processus itératif gouverné par la compatibilité entre la résistance du tube et son élasticité due à sa raideur propre et à sa fiche. Le dimensionnement structurel des pieux doit être réalisé avec un modèle (éléments finis ou logiciel spécifique) permettant de reproduire le comportement élasto-plastique du sol (Fascicule 62 Titre V). L'étude doit être menée à l'état limite ultime (ELU), avec des conditions de marnage, de courant, de houle et de vent extrêmes. Les états de mer considérés ont une période de retour centennale.

Une connaissance minimale du sol est donc indispensable à la conception de brise-lames flottants. Préalablement à toute étude, le Maître de l'ouvrage doit faire réaliser une campagne géotechnique permettant pour le moins de situer la position et l'évolution du toit du rocher, d'identifier et de caractériser les principales couches de sols. Le plan masse définitif établi, une seconde campagne doit permettre de déterminer les données géotechniques précises au droit de chaque ancrage.

\subsection{Efforts internes à la structure}

La solidité et la pérennité des pontons résultent essentiellement de l'application de principes de conception et de dispositions constructives saines et adaptées; ce qui n'est évidemment pas suffisant pour s'affranchir des études de détails complètes des structures. Pour faciliter la réalisation des brise-lames, les contraintes analysées ci-après doivent être intégrées dans la phase de conception.

\subsubsection{Structure des pontons}

Un brise-lames est formé de caissons en béton armé remplis de polystyrène haute densité qui en assure la flottabilité et l'insubmersibilité. Selon les dimensions liées à l'agitation et les coûts d'investissement consentis, la structure en béton des brise-lames peut être en cadre fermé ou, plus fréquemment, en cadre ouvert avec une protection de la sous face par un film synthétique. Les épaisseurs et volumes mis en œuvre résultent d'un compromis entre:

- la résistance de la structure aux efforts internes: épaisseurs minimales des voiles compatibles avec la mise en place de deux nappes d'armatures, volumes nécessaires pour reprendre les efforts transmis par les inserts aux liaisons,

- la flottaison du brise-lames, sachant que celle-ci doit satisfaire aux conditions d'atténuation des clapots (tirant d'eau), d'exploitation (franc bord) et de répartition intelligente des volumes (reprise des efforts de liaison).

L'assiette du brise-lames est ajustée, in fine, par le lestage de chambres insérées dans le polystyrène aux quatre angles de chaque flotteur. Compte tenu des tolérances sur les densités des matériaux et sur les épaisseurs mises en œuvre, l'étude de flottaison doit intégrer des "réserves" suffisantes pour obtenir, après lestage, l'assiette et le franc bord contractuels et nécessaires à l'exploitation.

\subsubsection{Dimensionnement des caissons}

La structure des caissons doit être justifiée vis à vis des sollicitations:

- globales: en intégrant les efforts dus à la houle, au vent et au courant,

- locales: en étudiant le fonctionnement transversal du caisson soumis aux variations du niveau d'eau et aux surcharges d'exploitation.

Toute section de béton doit être justifiée vis à vis de la fragilité en flexion locale comme en flexion globale. Conformément au règlement BAEL, les enrobages des parois minces peuvent être adaptés et réduits moyennant une composition particulière du béton qui doit présenter de très hautes caractéristiques mécaniques afin d'offrir une compacité suffisante pour protéger les armatures sans recourir à une protection cathodique complémentaire. Le respect de ces principes, issus des règles de l'art pour les ouvrages maritimes, et la mise en 
œuvre de dispositions constructives adaptées permettent d'assurer la pérennité du béton armé sur une très longue durée. L'étude du passage des efforts au béton armé par les inserts nécessite une attention toute particulière (colliers de guidage et liaisons entre pontons).

\subsubsection{Dimensionnement des colliers}

Les colliers de guidage sont constitués de structures métalliques protégées par une peinture anti-corrosion, munies de patins de glissement. Ils sont scellés dans le béton armé au moyen d'inserts boulonnés. Pour contrôler la raideur d'ancrage et adoucir les chocs, les colliers sont souvent munis de dispositifs semi-rigides constitués de deux défenses à cisaillement en néoprène fixées sur le caisson, directement ou par l'intermédiaire d'un support métallique. Une modélisation de la structure permet de justifier les profilés et leurs attaches, les scellements dans le béton, et de vérifier les contraintes dans les organes semi-rigides. Le déchirement du néoprène peut être évité en écrêtant, par des dispositifs adaptés, les sollicitations dépassant exceptionnellement les limites admissibles des défenses.

\subsubsection{Dimensionnement des liaisons semi-rigides}

Les pannes sont constituées d'éléments généralement réunis par des liaisons semi-rigides formées de deux défenses à cisaillement en néoprène précontraintes par une barre d'acier. Le fonctionnement des liaisons doit être soigneusement étudié pour que les sollicitations admissibles par les néoprènes ne soient pas dépassées. Des butées peuvent permettre d'écrêter les efforts extrêmes.

\section{Conclusion}

Les arguments développés ici rappellent que les brise-lames flottants sont avant tout des corps mobiles dont le comportement dynamique est autant gouverné par leurs modes propres que par l'excitation de la houle. Ils rappellent également qu'à la mer, l'accumulation d'expérience conduit à adopter des dispositions constructives saines, simples et adaptées, garantes d'efficacité et de longévité.

\section{Références}

1.Vugts J.H., 1968. International Shipbuilding Progress, Vol. 15. n67.

2.Bougis J., 1995. Analyse d'essais d'atténuateurs de houle, Rapport n94.011.

3.SOGREAH, 1984. Etude bibliographique sur les brise-clapots flottants.

4.STCPMVN, 1991. Conception des brise-clapots flottants, notice n ${ }^{\circ} 1.04$.

5.Blumberg G.P., Cox R.J., 1988. Floating Breakwater physical model testing for marina aplications, AIPCN, Bulletin $n^{\circ} 63$.

6.Bougis J., 2002. Etude d'extension du port de Nemours, Rapport n00.227.02. 